Casos Clínicos

Arch. Esp. Urol. 2010; 63 (6): 464-471

\title{
SARCOMA SINOVIAL RENAL EN EDAD TEMPRANA
}

Alfredo Ernesto Romero-Rojas, Julio Alexander DíazPérez, Oscar Alberto Messa-Botero y Fabián Enrique Neira-Mejia.

Instituto Nacional de Cancerología. Bogotá. Colombia. 'Departamento de Patología y Ciencias Forenses.

Universidad de Santander UDES. Bucaramanga. Colombia.

Resumen.- OBJETIVO: Describir un caso de Sarcoma Sinovial (SS) renal primario y realizar una revisión sobre este tema.

MÉTODO: Paciente masculino de 15 años de edad, con masa abdominal izquierda y pérdida de peso. A quien se documentaron por imágenes una lesión de $13 \mathrm{~cm}$ de diámetro, localizada en el polo inferior del riñón izquierdo, por lo cual, se realizó una biopsia renal, en la que se evidenció un tumor maligno indiferenciado, cuyos estudios de inmunohistoquimica sugerían los diagnósticos de tumor neuroectodermico primitivo Vs SS. Con lo anterior, iniciaron quimioterapia y realizaron nefrectomía radical. Actualmente el paciente presenta una sobrevida de 1.8 años.

\section{CORRESPONDENCIA}

Alfredo Ernesto Romero Rojas

Grupo de Patología

Instituto Nacional de Cancerolgía

Bogotá Colombia

aromero@cancer.gov.co

Aceptado para publicar: 12 de mayo 2009. 
RESULTADO: Se reconoció gran tumor renal izquierdo, de $22 \times 13 \times 12.5 \mathrm{~cm}$, multilobulado, con áreas de necrosis. Cuyo estudio histopatológico mostro una neoplasia maligna indiferenciada, compuesta por sabanas de células inmaduras. Los estudios de inmunohistoquimica permitieron concluir el diagnóstico de sarcoma sinovial renal pobremente diferenciado de variedad de células pequeñas. Estrategia de búsqueda de la literatura. Se realizó una búsqueda estructurada de la literatura, en las bases de datos Medline, Imbiomed y Scielo.

CONCLUSIÓN: Los SS de localización renal son extremadamente infrecuentes, con menos de 40 casos reportados, del cual se presenta el caso informado a edad más temprana. Estos tumores cuando se localizan en el riñón, representan un gran reto diagnóstico que requiere de la adecuada correlación clínico, imagenologica, quirúrgica, y patológica para su adecuado diagnóstico y manejo.

Palabras clave: Sarcoma sinovial. Riñón. Neoplasias del riñón. Sarcomas del riñón (Fuente: DeCS).

Summary.- OBJECTIVE: We report a primary renal Synovial Sarcoma (SS) case and analyze its features.

METHOD: A 15 year old male presented with left abdominal mass and weight loss. CT scan images showed a $13 \mathrm{~cm}$ mass located in the lower pole of the left kidney. Renal biopsy recognized an undifferentiated neoplasm, the immunohistochemistry suggesting the probability of neuroectodermic primitive tumor versus SS. Chemotherapy and radical nephrectomy were carried out. Pathological study showed a big multilobulated necrotic tumor $22 \times 13 \times 12.5 \mathrm{~cm}$. Histopathological study demonstrated a neoplasm composed by immature cells. Currently, patient has survived 1,8 years.

A structured bibliographical search was performed in the Medline, Imbiomed and Scielo databases.

RESULTS: The final immunohistochemistry studies gave the diagnosis of poorly differentiated renal SS small cell variety.

CONCLUSION: The renal SS is extremely infrequent, with less than 40 cases reported, of which this case reports the earlier age. These tumors, when located in the kidney, represent a great diagnostic challenge that requires adequate clinical, radiological, surgical, and pathological correlation for appropriate diagnosis and treatment.

Keywords: Synovial Sarcoma. Kidney. Neoplasias of the kidney. Sarcomas of the kidney (Source: MeSH).

\section{INTRODUCCIÓN}

Los sarcomas primarios del riñón son neoplasias raras que representan alrededor del 1 al $3 \%$ de los tumores malignos renales (1). El Sarcoma Sinovial (SS) es un tumor de partes blandas, clínica, genética y morfológicamente definido como de histogénesis incierta, que se origina predominantemente en las extremidades, cerca a la región para articular, capsula articular, bursas, y tendones $(1,2)$, en adolescentes y adultos jóvenes, de 15 a 40 años de edad, pero que puede localizarse en cualquier lugar del cuerpo (2), y en cualquier edad, afectando también diversas zonas sin relación aparente con las articulaciones (3). El representa el 5 al $10 \%$ de todos los tumores de partes blandas, y se caracteriza por presentar grados variables de diferenciación epitelial, con la presencia de una translocacion cromosomal especifica $t(X ; 18)(p 11.2 ; q 11.2)(3,4)$. Su nombre es atribuido a Sabrazes quien lo denominó de esta forma en 1934 (5), pero el primer reporte es atribuido a Lejars y Rubens-Duval, quienes lo denominaron endotelioma sinovial en 1919, ya que ellos realizaron la primera descripción completa de esta entidad, formulando sus hallazgos morfológicos típicos, aunque también plantearon un origen en la sinovia articular (6), lo cual nunca fue comprobado y actualmente se considera refutado $(2,3,7)$; un trabajo similar fue desarrollado por Smith en 1927 (8). La presentación de este tumor ha sido informada en diversas regiones y órganos, aunque de forma muy infrecuente, con reportes en cabeza y cuello, tórax, pared abdominal y reproperitoneo; y casos extremadamente inusuales de localización en vulva, pene, próstata, pleura, pulmón, corazón, esófago, estomago y riñón (9-12). El SS renal es extremadamente raro, del cual se han reportado 26 casos en la literatura, encontrando en todos ellos un pobre pronóstico $(1-3,7,9,13)$. La primera descripción de esta inusual localización la realizó Arnold quien describió un sarcoma embrionario renal en 1995, y posteriormente, Argani y colaboradores, en 2000 , anunciaron que estos tumores correspondían a la contraparte renal de los SS de partes blandas (13), luego de estudiar un grupo de casos diagnosticados como Sarcomas embrionarios renales, en el cual identificaron 15 casos de SS renal primario, 4 de ellos confirmados por técnicas de citogenética, aunque ya Faria en 1999 había enunciado la posibilidad de que casos de Sarcomas embrionarios renales se tratasen de SS (14). En este trabajo se describirá un caso de SS primario renal y realizara una revisión sobre este tema.

\section{CASO CLÍNICO}

Paciente masculino de 15 años de edad, quien consultó por masa abdominal izquierda, con pérdida de $8 \mathrm{~kg}$ de peso en 6 meses, además de sudoración nocturna, sin otros síntomas asociados. Se realizó una Tomografía Axial Computarizada (TAC) abdominal que mostró masa de $13 \mathrm{~cm}$ de diámetro localizada en el polo inferior del riñón izquierdo, la cual evidenciaba gran trom- 
bo tumoral en la vena cava que se extendía hasta aurícula izquierda, con riñón contralateral de aspecto usual (Figura 1). Con los anteriores datos, se decidió realizar biopsia renal por punción con aguja gruesa, en la cual se evidenció un tumor maligno indiferenciado, extensamente necrosado, cuyo estudio con tinciones de inmunohistoquimica fue positivo para vimentina, BCL2 y CD99, que sugería los diagnósticos de tumor neuroectodermico primitivo Vs sarcoma sinovial renal. Posteriormente, se administraron 3 ciclos de quimioterapia prequirugica con vincristina, y luego se realizó nefrectomía radical, en la cual se encontró gran tumor en polo renal inferior, el cual es disecado y extraído incompletamente, por la presencia de trombo tumoral macroscópico. El paciente continúo con vincristina, doxurubicina, isofosfamida y etoposido, además de radioterapia en el hemiabdomen anterior y posterior. Luego de un seguimiento de 1 año el paciente presento metástasis documentada histopatologicamente en lóbulo pulmonar izquierdo y actualmente se encuentra en manejo paliativo, con una sobrevida actual total de 1.8 años.
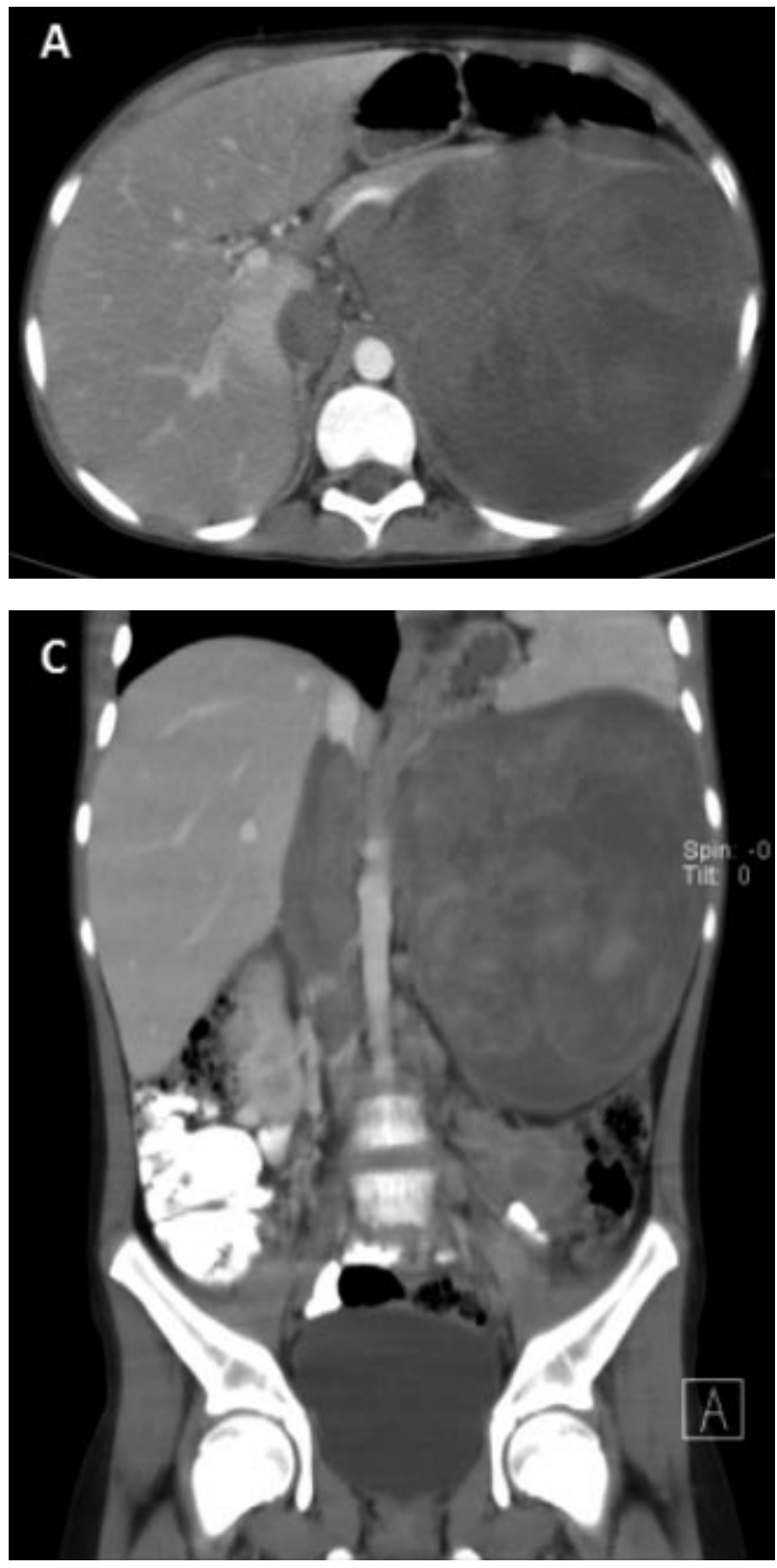
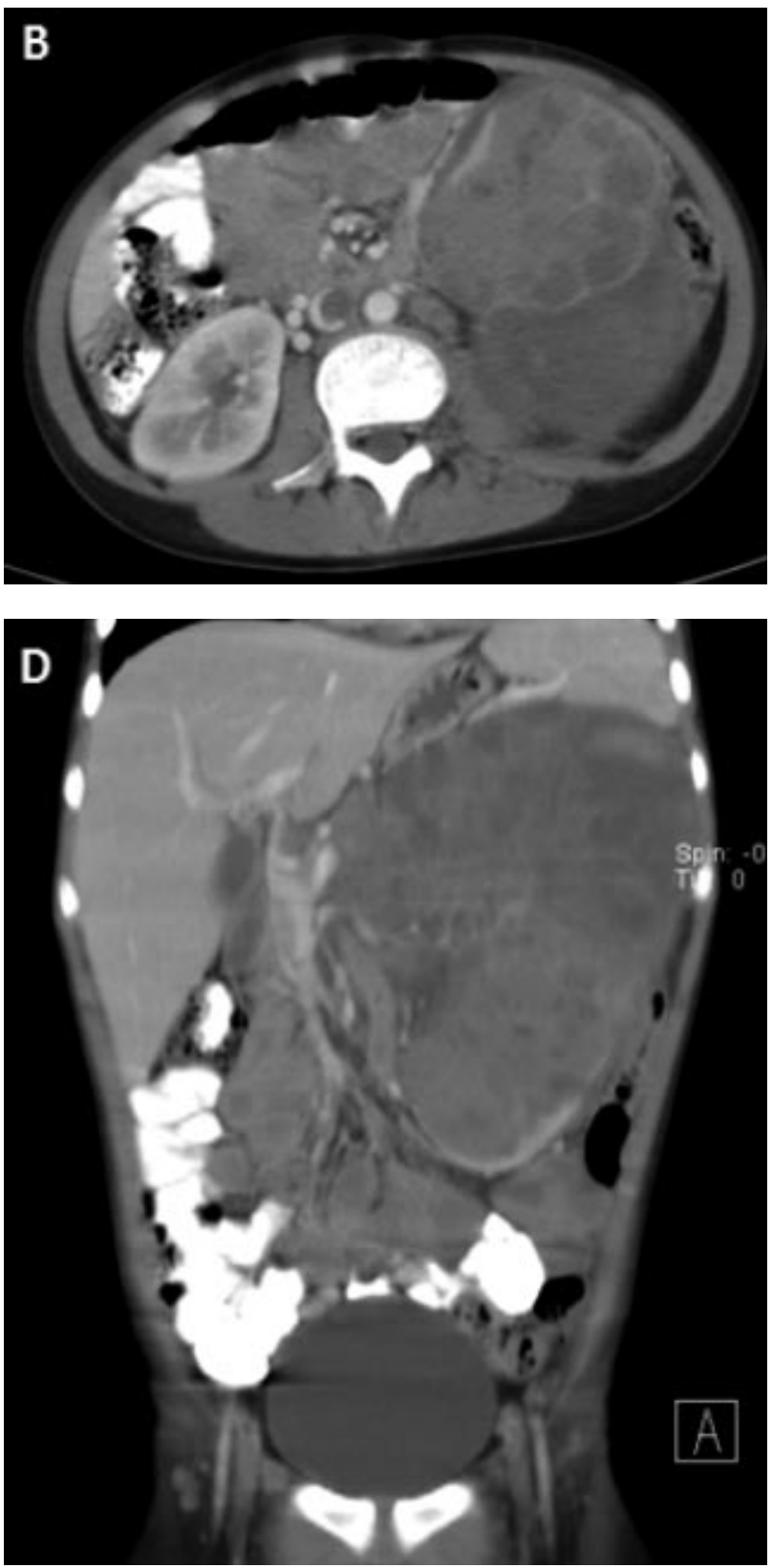

FIGURA 1. En A se reconoce gran masa que infiltra y ocupa la fosa renal izquierda (Corte axiall. Observando en B cortical renal inferior deformada y trombo tumoral en la vena cava inferior (Corte axial). En C y D se identifica masa que infiltra y ocupa la fosa renal izquierda, deforma la cortical renal inferior, y extenso trombo tumoral en la vena cava inferior 


\section{Estudio Anatomopatológico}

En el servicio de patología se recibió riñón izquierdo, que midió $22 \times 13 \times 12.5 \mathrm{~cm}$, con uréter de $3.8 \times 0.7 \mathrm{~cm}$. Cuya superficie externa era multilobulada e irregular. Al corte se reconoció gran masa de aspecto carnoso, pardo oscuro, muy friable que comprometía todo el parénquima renal, el seno renal y se extendía al hilio renal. El estudio histopatológico mostro riñón comprometido por neoplasia maligna indiferenciada, compuesta por sabanas de células inmaduras de núcleos ovales, irregulares, rodeados por un citoplasma eosinófilo claro, con extensas zonas de necrosis (Figura 2), la cual comprometía los tejidos blandos perirenales, la glándula suprarrenal y borde de sección vascular (vena renal). No se observó diferenciación epitelial, ni compromiso del borde de uréter. Las tinciones de inmunohistoquimica (Tabla I para conocer sus características relevantes) mostraron reactividad en las células tumorales para vimentina, calponina, FLI 1, CD99, BCL2, EMA y $A E 1 / A E 3$; con negatividad para cromogranina, sinaptofisina, WT1, enolasa neuronal especifica, CD34, desmina, MYOD y LCA (CD45) (Figura 3). Con lo anterior, se realizó el diagnóstico de sarcoma sinovial pobremente diferenciado de variedad de células pequeñas.

\section{Estrategia de búsqueda de la literatura}

Se realizó una búsqueda estructurada de la literatura, con base en la metodología Cochrane, en las bases de datos Medline, Imbiomed y Scielo, utilizando las palabras clave "Sinovial sarcoma" y "kidney" desde el año 1960 a Marzo de 2009, limitando la búsqueda a artículos originales escritos en idioma inglés y español, la metodología empleada se muestra en la Tabla 2. De los artículos encontrados, se seleccionaron los considerados relevantes por los autores.

\section{DISCUSIÓN}

Los sarcomas originados en el riñón son neoplasias muy infrecuentes (2), ellos son clasificados en base a su tipo histológico en leiomiosarcoma, rabdomiosorcoma, liposarcoma, fibrosarcoma, angiosarcoma, liposarcoma, histiocitoma fibroso maligno, hemangiopericitoma, condrosarcoma, osteosarcoma y SS $(2,3)$. El más común de estos tumores es el leiomiosarcoma el cual representa el 40 al $60 \%$ de todos los casos reportados de sarcoma renal. El SS renal es por mucho una de las neoplasias más infrecuentes del riñón, con solo 26 casos encontra-
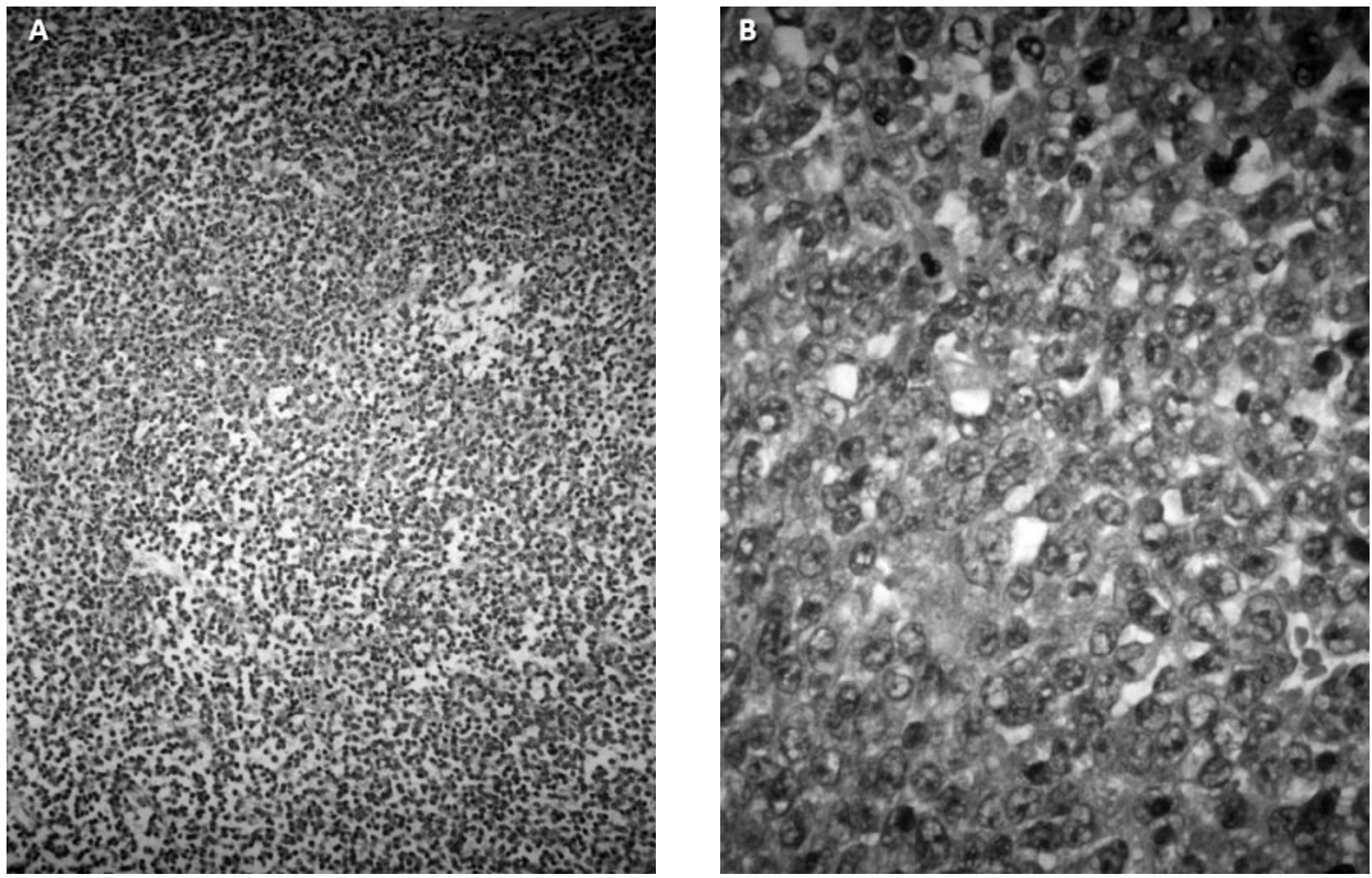

FIGURA 2. El estudio histopatológico mostró remplazo completo del riñón por neoplasia maligna indiferenciada, compuesta por gran cantidad de células de tamaño pequeño, de núcleos ovales, irregulares, con frecuentes mitosis atípicas, rodeados por un citoplasma eosinófilo claro, dispuestas en sabanas (A y B, HE 5 y 40x). 
dos en nuestra búsqueda. El origen de estos tumores no es bien comprendido, por lo cual se les agrupa dentro de un grupo heterogéneo de neoplasias denominado tumores malignos de tipo incierto, ya que no se asemejan a los tejidos normales del organismo, aunque algunos autores consideran que se derivan de células mesenquimales con diferenciación epitelial $(1,2)$.

Los SS renales se presentan en edades entre los 19 y los 71 años, con una media de presentación a los 38 años
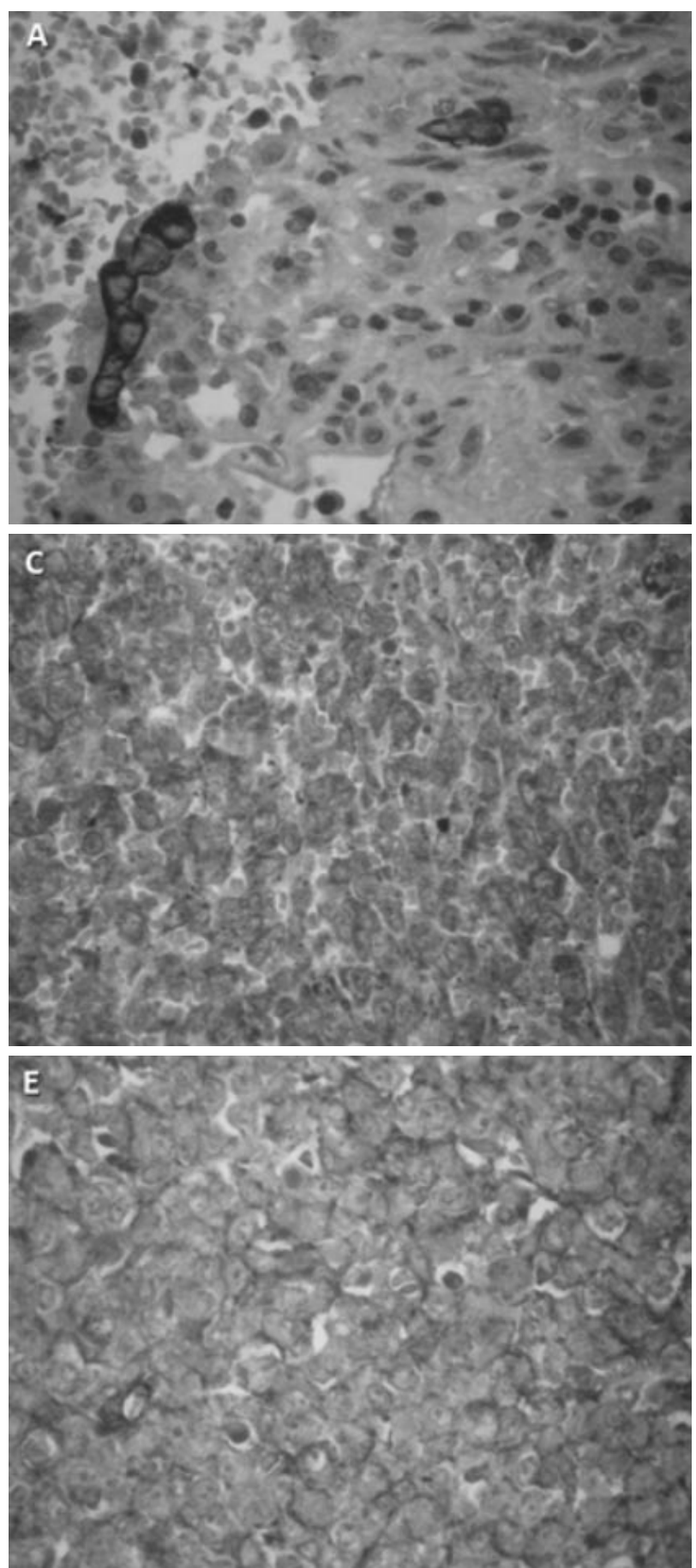

de edad $(3,9)$, por lo cual nuestro caso se constituiría en el reporte a edad más temprana. Estos tumores poseen una preponderancia por la afección al género masculino (9). Los síntomas clínicos más frecuentemente observados son similares a los evidenciados en otros tumores renales malignos, lo cuales incluyen el dolor abdominal y hematuria, como los mas referidos (7).

Macroscópicamente los SS renales se observan como grandes masas que usualmente miden entre 5 y $20 \mathrm{~cm}$
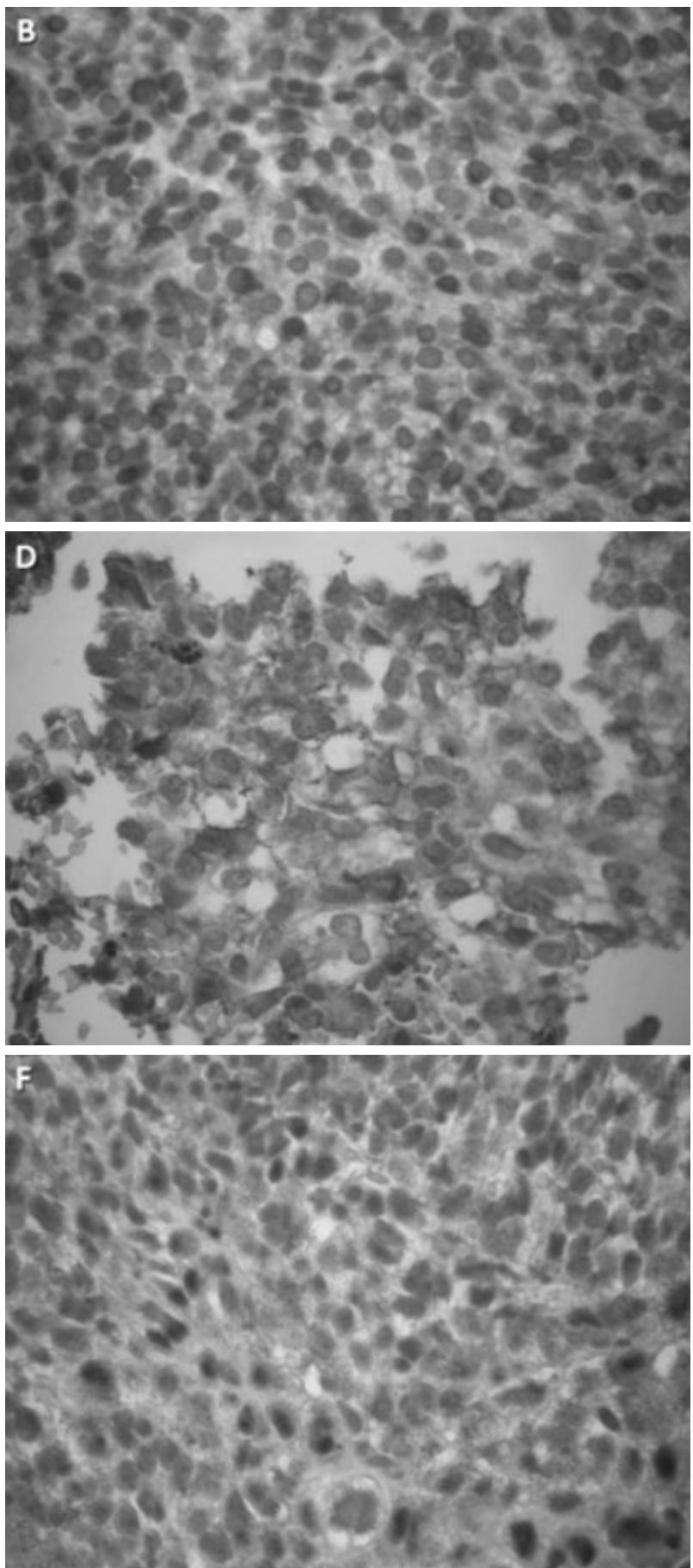

FIGURA 3. En las tinciones de inmunohistoquimica se evidenció reactividad en las células tumorales para AE I/AE3 (A), BCL2 (B), calponina (C), CD99 (D), EMA (E), y Fll7 (F). (Técnica de Adivina Biotina 40x). 
TABLA I. ESTRATEGIA DE BÚSQUEDA DE LA LITERATURA, SE UTILIZAN DESCRIPTORES MESH PARA LA BASE DE

DATOS MEDLINE, PARA LAS DEMÁS BASES DE DATOS

LA BÚSQUEDA SE REALIZÓ EN FORMA ANÁLOGA.

\begin{tabular}{|c|c|c|}
\hline Paso & Descriptor (Palabra Clave) & № de Citaciones \\
\hline 1 & Synovial Sarcoma & 2733 \\
\hline 2 & Kidney & 561166 \\
\hline 3 & 1 AND 2 & 77 \\
\hline 4 & Limits & 26 \\
\hline
\end{tabular}

de diámetro $(7,9)$, en los que ocasionalmente se encuentran quistes asociados, y en un porcentaje menor el compromiso de estructuras adyacentes y de los grandes vasos renales $(9,10)$. Si se encuentran lesiones de crecimiento lento se observan como tumores lobulados, bien delimitados y recubiertos por una pseudocapsula de tejido conectivo. Contrario a las lesiones de rápido crecimiento las cuales se observan desorganizadas, con grandes zonas de necrosis (10).

Histopatológicamente, los SS renales siguen la misma configuración y clasificación de los SS de otras localizaciones. Ellos se han clasificado en tres subtipos, monofásicos, bifásicos y pobremente diferenciados, dependiendo de sus componentes celulares. El tipo bifásico está caracterizado por la presencia de una mezcla de células epiteliales y mesenquimales malignas, fácilmente reconocibles, las cuales pueden estar presentes en proporciones diferentes, las células epiteliales con frecuencia forman elementos glandulares, las cuales suelen contener secreciones eosinofilicas homogéneas en su interior (10). El tipo monofásico está compuesto únicamente por células epiteliales o fusiformes, lo cual dificulta su diferenciación con otros sarcomas como los fibrosarcomas, hemangiopericitomas o fumores malignos de la vaina del nervio periférico, lo que hace imprescindible para su definición la utilización de tinciones de inmunohistoquimica (15) ya que con ellas se logra la diferenciación

TABLA II. CARACTERISTICAS DEL PANEL DE INMUNOHISTOQUÍMICA EMPLEADO EN EL ESTUDIO DEL CASO.

\begin{tabular}{|c|c|c|c|}
\hline Marcador & Clon & Dilución & Casa Comercial \\
\hline Queratinas & AE1AE3 & $1: 100$ & DAKO \\
\hline BCL2 & Bcl2/100/D5 & $1: 50$ & Novocastra \\
\hline Cromogranina & DAK-A3 & $1: 100$ & Dako Cytomation \\
\hline EMA & E29 & $1: 50$ & Dako Cytomation \\
\hline Calponina & CALP & $1: 500$ & Dako Cytomation \\
\hline CD99 & HO36-11 & $1: 100$ & Neomarkers \\
\hline Ki67 & MIB1 & $1: 100$ & Dako Cytomation \\
\hline S100 & 4C4.9 & $1: 50$ & Neomarkers \\
\hline Vimentina & V-9 & $1: 200$ & BioGenex \\
\hline Sinaptofisina & SNP88 & $1: 100$ & BioGenex \\
\hline FLI1 & FLI1 & Prediluido & Thermo Scientific \\
\hline WT1 & WT49 & $1: 49$ & Novocastra \\
\hline BES/NC & $1: 100$ & Dako Cytomation \\
\hline Enolasa Neuronal Especifica & QBEND & $1: 50$ & BioGenex \\
\hline CD34 & S.8A & $1: 50$ & Dako Cytomation \\
\hline MYOD & PD7/26/16 & $1: 50$ & BioGenex \\
\hline LCA & HO36-1.1 & $1: 100$ & Thermo Scientific \\
\hline CD99/MIC2 & & & \\
\hline
\end{tabular}


de otros tumores $(15,16)$. Finalmente, el tipo pobremente diferenciado también ha sido reportado en localización renal, este tipo aporta cerca del $20 \%$ de los casos de SS renal (7), asociándose a un pésimo pronostico, además en el estudio de este ultimo tipo de SS se debe subdividir en 3 categorías: 1. una variante de células grandes que tiene características intermedias entre células fusiformes y epitelioides con ocasionales elementos rabdoides, 2. una variante de células pequeñas muy similar a un sarcoma de Ewing/tumor neuroectodermico primitivo, como nuestro caso, y 3. una variante fusocelular de alto grado que semeja otras lesiones de alto grado como fibrosarcomas y tumores malignos de la vaina del nervio periférico. Estas lesiones a pesar de su agresividad pueden tener un conteo mitótico bajo, lo que dificulta aun mas su adecuado diagnóstico. A pesar de esta configuración bastante inespecífica, los SS poseen dos características que pueden ayudar a su diferenciación de otras lesiones, la primera de ellas es la mezcla de áreas celulares con zonas hipocelulares que muestran hialinización, degeneración mixoide o calcificación, la segunda es la presencia de mastocitos que son más numerosos en las áreas de células fusiformes $(17,18)$.

Los SS renales al igual que los localizados en otras zonas del cuerpo muestran positividad para el antígeno epitelial de membrana (EMA, del ingles Epithelial Membrane Antigen) hasta en el $97 \%$ de los casos, y para citoqueratinas en el $69 \%$. Porcentajes que son menores en los SS poco diferenciados $(15,16)$. Estos tumores también son positivos para CD 99 en el 60 al $70 \%$ de los casos, proteína $\mathrm{BCL} 2$ se expresa de manera difusa en la casi totalidad de los SS, proteína FLI1, y proteína S-100 (13). Además se ha reportado que la calponina es un marcador útil en la identificación de los SS de tipo pobremente diferenciado, en el cual se han documentado una positividad que varía entre el 29 y el $81 \%(19,20)$.

En la totalidad de los sarcomas sinoviales, independiente de su tipo y localización, muestran la translocación $\mathrm{t}(\mathrm{X} ; 18)(\mathrm{p} 11 ; \mathrm{q} 11)(1,4,12)$. Esta translocación implica la fusión del gen SYT del cromosoma 18 con los genes SSX1 o SSX2 del cromosoma X (los dos en Xp 1 1) o con menos frecuencia con el gen SSX4. Esta alteración puede determinarse con la reacción en cadena de la polimerasa transcriptasa inversa (RT-PCR) o con hibridización in situ con fluorescencia (FISH), utilizando tejido fresco o incluido en parafina $(1,21,22)$.

Los SS se deben estudiar, al igual que los demás sarcomas renales, siguiendo el sistema de estadiaje TNM, en este caso en particular, su conocimiento es de mayor utilidad, ya que debido a la presencia del gran trombo tumoral, el estadio aumenta de un T2 a un T3b aumentado el estadio total de estadio II a estadio III.

La base del tratamiento de los SS como de otros sarcomas renales es la nefrectomía radical, la cual debe proveer márgenes libres de tumor, para considerarse exitosa $(2,3)$. Pero esto no se logra en todos los pacien- tes ya que el 30 al $50 \%$ de estos no tienen márgenes adecuados, lo cual se relaciona con el desarrollo de metástasis a distancia, empobreciendo así el pronóstico $(7,23)$. La radioterapia actualmente no se usa en forma rutinaria, aunque anteriormente era pieza fundamental en el manejo de estas lesiones, donde se había usado desde 1902 (7). En el momento, solo se le considera a la quimioterapia como una medida útil en el manejo de estas neoplasias, esta se realiza generalmente con doxurubicina e ifosfamida, ya que logra aumentar el tiempo de sobrevida de los pacientes afectados por SS. En este tumor también se han utilizado esquemas de rescate con altas dosis de doxurubicina e ifosfamida combinadas con etoposido y cisplatino, con las que se ha logrado estabilizar la enfermedad durante tiempos prolongados $(2,7)$.

\section{CONCLUSIÓN}

Presentamos un caso de SS de localización renal, la cual es una de sus presentaciones menos comunes, este caso tiene la particularidad de ser el informado a una edad más temprana. Estos tumores cuando se localizan en el riñón representan un gran reto diagnóstico que requiere de la adecuada correlación clínico, quirúrgica, y patológica para su adecuada definición y manejo.

\section{BIBLIOGRAFIA y LECTURAS RECOMENDADAS ( ${ }^{*}$ lectura de interés $y^{* *}$ lectura fundamental)}

**1. Koyama S, Morimitsu Y, Morokuma F, Hashimoto H. Primary synovial sarcoma of the kidney: Report of a case confirmed by molecular detection of the SYTSSX2 fusion transcripts. Pathol Int, 2001;51(5):385-91.

**2. Divetia M, Karpate A, Basak R, Desai SB. Synovial sarcoma of the kidney. Ann Diagn Pathol. 2008;12(5):333-9.

3. Perlmutter AE, Saunders SE, Zaslau S, Chang WW, Farivar-Mohseni H. Primary synovial sarcoma of the kidney. Int J Urol. 2005;12(8):760-2.

4. Clark J, Rocques PJ, Crew AJ. Identification of novel genes, SYT and SSX, involved in the $\mathrm{t}(\mathrm{X}: 18)$ (p11.2;q11.2) translocation found in human synovial sarcoma. Nat. Genet. 1994; 7: 502-508.

5. Sabrazes J, Loubat E, de Grailly R. Synovial sarcomes. Gaz Hebd Sc Med Bordeaux. 1934;55:754-62.

6. Lejars M, Rubens-Duval M. Les sarcomes primitifs des synoviales articulaires. Rev Chir. 1910;41:751-83.

**7. Park SJ, Kim HK, Kim CK, Park SK, Go ES, Kim $\mathrm{ME}$, et al. A case of renal synovial sarcoma: complete remission was induced by chemotherapy with doxorubicin and ifosfamide. Korean J Intern Med. 2004;19(1):62-5.

8. Smith LW. Synoviomata. Am J Pathol. 1927;3:355368.

9. Paláu L MA, Thu Pham T, Barnard N, Merino MJ. 
Primary synovial sarcoma of the kidney with rhabdoid features. Int J Surg Pathol. 2007;15(4):421-8.

10. Cihak RA, Lydiatt WM, Lydiatt DD, Bridge JA. Synovial sarcoma of the head and neck: chromosomal translation $(\mathrm{X} ; 18)$ as a diagnostic aid. Head Neck 1997; 19: 549-553.

11. Dei Tos AP, Dal Cin P, Sciot R, Furlanetto A, Da Mosto MC, Giannini C, et al. Synovial sarcoma of the larynx and hypopharynx. Ann. Otol. Rhinol. Laryngol. 1998; 107: 1080-5.

12. Iwasaki $H$, Ishiguro $M$, Ohjimi $Y$, Ikegami $H$, Takeuchi T, Kikuchi M, et al. Synovial sarcoma of the prostate with $\mathrm{t}(\mathrm{X} ; 18)(\mathrm{p} 11.2 ; \mathrm{q} 11.2)$. Am. J. Surg. Pathol. 1999; 23: 220-6.

13. Argani P, Faria P, Epstain JI, Reuter VE, Beckwith B, Ladanyi M. Primary renal synovial sarcoma: molecular and morphologic delineation of an entity previously included among embryonal renal sarcoma. Am. J. Surg. Pathol. 2000; 24: 1087-96.

14. Faria P, Argani P, Epstein JI, Reuter VE, Beckwith B. Primary synovial sarcoma of the kidney: A molecular reappraisal of a subset of so-called embryonal renal sarcoma (Abstract). Mod. Pathol. 1999; 12: 94A.

15. Folpe AL, Schmidt RA, Chapman D, Gown AM. Poorly differentiated synovial sarcoma: immunohistochemical distinction from primitive neuroectodermal tumors and high-grade malignant peripheral nerve sheath tumors. Am J Surg Pathol 1998; 22:673.

16. Olsen SH, Thomas DG, Lucas DR. Cluster analysis of immunohistochemical profiles in synovial sarcoma, malignant peripheral nerve sheath tumor, and Ewing sarcoma. Mod Pathol 2006; 19:659.

17. Winnepenninckx V, De Vos R, Debiec-Rychter M, Samson I, Brys P, Hagemeijer A, et al. Calcifying/ ossifying synovial sarcoma shows $t(X ; 18)$ with SSX2 involvement and mitocondrial calcifications. Histopathology 2001; 38:141.

*18. Krane JF, Bretoni F, Fletcher CD. Myxoid synovial sarcoma: an underappreciated morphologic subset. Mod Pathol 1999; 12:456.

*19. Fisher C, Montgomery E, Healy V. Calponin and h-caldesmon expression in synovial sarcoma; the use of calponin in diagnosis. Histopahology 2003; 42:588.

*20. Ono H, Yoshikawa H, Ueda T, Yamamura H, Kudawara I, Manou M, et al. Expression of smooth muscle calponin in synovial sarcoma. Sarcoma. 1999;3(2):107-13.

*21. Mirza M, Zamilpa I, Bunning J. Primary renal synovial sarcoma. Urology. 2008;72(3):716.e11-2.

22. Gabilondo F, Rodríguez F, Mohar A, Nuovo GJ, Domínguez-Malagón H. Primary synovial sarcoma of the kidney: corroboration with in situ polymerase chain reaction. Ann Diagn Pathol. 2008;12(2):134-7.

**23. Kim DH, Sohn JH, Lee MC, Lee G, Yoon GS, Hashimoto $\mathrm{H}$, et al. Primary synovial sarcoma of the kidney. Am J Surg Pathol. 2000;24(8):1097-104. 